\title{
Research on the Design of Safety Education Identification System for Art College Laboratories
}

\author{
Lingtao Liu $^{1, *}$ \\ ${ }^{1}$ Experimental Teaching Center, Sichuan Fine Arts Institute, Chongqing, China \\ "Corresponding author. Email: 734557916@qq.com
}

\begin{abstract}
This article focuses on the laboratory safety education courses, takes the changes in the learning style of college students in the era of picture-reading as the foothold, analyzes the status quo and problems of laboratory safety education courses in colleges and universities in recent years, and builds a multi-disciplinary integrated laboratory safety education course. It also introduces how to use the means of identification to realize the effective path of course content, teaching methods and assessment methods. The teaching method that uses identification as the main content and text as the supplement can increase the visibility of the course content, improve the teaching effect, adapt to the way contemporary college students acquire knowledge, continuously improve their safety awareness, enhance their ability to operate safely and respond to sudden accidents, form good laboratory safety operation habits, effectively reduce laboratory safety risks, and provide reference for college laboratory safety education courses.
\end{abstract}

Keywords: Laboratory, Safety education, Identification design.

\section{INTRODUCTION}

The Experimental Teaching Center of Sichuan Fine Arts Institute (National Experimental Teaching Demonstration Center), which will be hereinafter referred to as "the Center", is the first batch of national art experimental centers established by the Ministry of Education in 2008, and is the leading unit of the Art Group of the National Demonstration Center for Experimental Education. The Center has 19 specialized laboratories (including Clothing Laboratory, Fiber Laboratory, Printmaking Laboratory, Ceramic Laboratory, 3D Printing Laboratory, Virtual Studio, Metal Processing Laboratory, Lacquer Laboratory, Architectural Physics Laboratory, Architectural Model Laboratory, Clay Model Laboratory, Photography and Video Recording Laboratory, Woodworking Laboratory, Hard Material Laboratory, Glass Laboratory, Clay Sculpture Lofting Laboratory, Comprehensive Materials Laboratory, Text Activation Laboratory, and Precision Machining Laboratory), serving four

*Fund: The Project of "Research on the Construction of Laboratory Safety Education Curriculum in Art Colleges and Universities" from Sichuan Fine Arts Institute, with the project number of 5142047 . subject teaching of fine arts, design, opera and film and television, and landscape architecture. The Center is open to teachers and students of the whole school, offering a total of 211 experimental courses. In addition to the experimental courses of various departments, it also satisfies students' daily creation and graduation creation, and cooperates with teaching and scientific research, teaching experiment and other teaching work. The Center also undertakes relevant projects of enterprises and public institutions in the province, making contributions to the development of local economy and culture.

College laboratory is an important place for practical teaching and innovative talent training in universities, as well as an important base for the construction of "double first-rate" [1]. Nowadays, with the transformation of information technology, visual culture and media culture continue to impact, college students have more diversified ways of acquiring knowledge, and the text learning method no longer has advantages [2]. In addition, college students have stronger ability to accept new things, and the learning of identification has become the main learning method. Therefore, laboratory teaching using identifications will be a teaching 
exploration and attempt that is more in line with the characteristics of the era of picture-reading. Laboratory identification teaching refers to the important content and practical application of screening, integrating, selecting, and transferring the safety knowledge of experimental safety courses through generalizing images in the process of teaching, which is more in line with the aesthetic needs of contemporary college students, so as to achieve a serious and lively teaching effect.

\section{THE NECESSITY FOR THE CONSTRUCTION OF LABORATORY SAFETY EDUCATION COURSE IN ART COLLEGES}

In recent years, laboratory safety accidents in colleges and universities across the country have occurred from time to time. How to improve the safety education level of college laboratories has become a top priority for college laboratories. Many colleges and universities have changed laboratory safety training to compulsory laboratory safety education courses, including the combination of online education and offline education, as well as the combination of virtual reality and real scene teaching [3]. It can be seen that the management of laboratories in colleges and universities has changed from the perspective of technical specifications to systematic learning of safety knowledge. The laboratory safety education course of Sichuan Fine Arts Institute is different from other comprehensive universities to a certain extent in the following aspects: First, the experiment content of art colleges is mainly physical reaction, supplemented by chemical reaction, mostly using mechanical equipment for shaping and imagemaking, while a few laboratories use chemical materials such as acid and alkali to create images through corrosion. However, the use of chemical materials is not standardized and there are potential safety hazards. Second, there are many flammable materials stored in fine art colleges, such as wood, paper, turpentine, etc. The storage of materials is relatively difficult and the space requirement is greater. Third, the students of fine art colleges have weaker logical thinking but strong imaginal thinking and operational ability, causing them to pay insufficient attention to text-based rules and regulations and operating specifications in experiments, laying hidden dangers to laboratory safety. Fourth, fine art creation pursues individualized artistic creation. In order to achieve artistic effect, students often perform violation operations in the laboratory, such as privately refitting small instruments and mixing different painting materials. Only by conducting effective safety education on the specific problems of the above-mentioned fine art college laboratories can it be possible to avoid the occurrence of laboratory safety accidents and escort students for various artistic creations.

\section{CURRENT STATUS AND PROBLEMS OF LABORATORY SAFETY EDUCATION COURSES}

\subsection{The Institutional Learning Is Boring}

Susan Sontag said in "On Photography": "Photo is not only an image, but also an interpretation of reality; it is also a trace; it is something directly imprinted from reality...." [4] As colleges and universities attach great importance to laboratory safety work, the laboratory management system, rules and regulations, operating instructions, and codes of conduct of student have been continuously improved. Various rules and regulations are posted on the wall as required, but few students will stop and watch and understand them in detail. In the previous experiment safety education courses, it was difficult for laboratory managerial staff to explain various rules and regulations, they could only repeat the instructions, and the safety education was not in place. In the end, the teacher repeated, and the students didn't like to listen, which caused an embarrassing situation where an autumn breeze passed the ear, and the various rules and regulations posted on the wall turned into a mere formality. Therefore, students couldn't be attracted by the content of the course at the beginning of the safety course and failed to effectively enter the learning state. And in the actual experiment process, there were omissions of safety knowledge points, leaving latent dangers of accidents for laboratory safety.

\subsection{Miscellaneous Content and Rote Memory}

With the updating of technology and the change of equipment, laboratories continue to introduce new equipment and new experimental methods, and the content of experiment safety education courses is becoming more and more diversified and systematic. It includes laboratory rules and regulations, laboratory space cognition, equipment application methods, operating specifications, 
emergency plans and emergency responses, etc. The laboratory safety course is a complex comprehensive course, there are many knowledge points involved in each part of it, and each knowledge point requires students to understand and master. Due to the complexity of course knowledge, students lack enthusiasm and initiative in learning, and passively accept the content of the course. Learning by rote memorization is harmful for students to achieve learning effect and they are likely to forget.

\subsection{The Model Is Outdated and Lacks Features}

The knowledge learning of traditional safety education courses relies mainly on teachers' teaching, and students passively accept it. From a subjective point of view, this places a higher demand on teachers' mastery of safety skills. Teachers must continuously learn new knowledge and new methods in order to keep up with the management needs of the studio. Objectively speaking, in the course, students rely too much on the teacher's teaching, passively accept the content of the course, lack active thinking and speculation, cannot actively participate in the course, and lack the interaction with the teacher. The teacher is not clear about the students' learning situations, and the students have a smattering of the knowledge they have learned.

\subsection{Single Assessment and Lack of Participation}

During the course, the assessment method only pays attention to the assessment of the results and ignores the assessment of the process. This method of assessment will cause students to focus on the content of the exam. Before the exam, many students hope that the teacher will give the scope for them to review, and then, they will stay up and recite all night, make effort at the last moment, and finally make short shrift of the exam, which won't be conducive to the improvement of students' awareness of experiment safety. In the students' cognition, laboratory safety education courses are supplementary courses for experiments, and whether the experiment results can be achieved is the purpose of course learning. Therefore, in class, students take no count of this course, and cannot correctly recognize the influence of experiment methods on experiment results, leaving safety hazards to the laboratory.

\section{ANALYSIS OF THE CONSTRUCTION PATH OF LABORATORY SAFETY COURSE IDENTIFICATION SYSTEM}

\subsection{Prohibited Identification System}

Text is abstraction and generalization of things, which is abstract. Visualizing the text content of abstract rules and regulations is a process from abstraction to perceptual intuition, which shortens the time for learning knowledge and obtains a large amount of knowledge content in a limited time [5]. The combination of image and text is not to ignore the narrative of the text, but to combine the narrativity of the text with the visibility of the image. The two bring out the best in each other and complement each other, which can not only explain the text better, but also enhance students' interest in reading. Whether it is the narrativity of the text or the visibility of the image, the ultimate goal is to convey knowledge [6]. Affected by modern information technology, image has become an important way for contemporary college students to acquire knowledge and communicate. Popularizing the theoretical system specifications makes the content of the experiment safety education course more vivid and interesting, which is in line with the way students acquire knowledge. This method will make the originally boring rules and regulations teaching become vivid and colorful, not only spreading knowledge, but also bringing pleasing artistic experience to students.

\subsection{Operation Specification Identification System}

Image is the basis of human vision, an objective reflection of natural scene, and an important source for human beings to understand the world and human beings [7]. The purpose of using images is to restore objective reality. The use of identifications can allow students to intuitively understand the basic attributes of the laboratory. In the laboratory safety courses, there are many applications of identifications: 1. Before entering the laboratory, students need to know the space division and the function introduction of each area of the laboratory in advance. Using images, they can intuitively see the space layout and functions of the laboratory and have a certain understanding of the laboratory. 2. Teachers should explain the cognition and precautions of equipment and chemical reagents in the laboratory as well as the 
attribute of each material. Using images, students can recognize and know the equipment, and learn to use the equipment in theory, providing theoretical support for the formal use of the equipment. 3 . Each item in the laboratory has its corresponding placement area and fixed position. The use of images can allow students to clearly understand the specific placement of each item. Placing pictures on specific locations of items can enhance students' intuitive experience. 4. Teachers should use pictures in emergency plans and emergency measures to equip students with emergency responses and means of escape. In the use of images, images are not only used for images. Before entering the laboratory, students have a preliminary understanding of the laboratory in their minds. Teachers need to continue to explain the operation specifications and the use of equipment in the specific operation links, discover students' problems during the experiment, and ask students to correct them immediately through continuous supervision, so as to form students' good experiment habits.

\subsection{Logo System for the Decomposition of Experiment Steps}

The diagrammatized experiment step simplifies the complexity of each experiment process to a vivid and concrete display. Diagram refers to the use of graphics to analyze or calculate and explain the reason. 1. The experiment steps of logo decomposition are to set an experiment step as an identification group, form a closed operation process through identification for an operation specification, and decompose each operation detail into several identifications. Each piece of identification shows step-by-step actions and uses images of real scenes to restore the scene, allowing students to obtain information in a short period of time, which has the effect of simplifying complexity and achieving the purpose of virtual reality. 2. The experiment steps of logo decomposition can also accurately locate the experiment effect, so that students can grasp the corresponding knowledge in order to meet the experiment requirements. 3. Teachers answer the universal and specific problems in experiment teaching, and analyze and answer one by one from the causes of the problems and the key steps that will lead to such problems. This process starts from assumptions and is reasoned based on assumptions.

\subsection{Combining the In-class Assessment of Talking About Pictures and the End- of-course Assessment}

The assessment method of this course is divided into two parts. The first part is the assessment in class, accounting for $40 \%$ of the course grade. With the way of talking about pictures, firstly, the teacher teaches and demonstrates, then, the students imitate the teacher's teaching method and make comments based on the image content provided by the teacher, which will mobilize the students to discover problems and actively think, make them follow the teacher's ideas at any time, arouse their enthusiasm, make them change from passive listening to active thinking, and enhance the interactivity of the course. The second part is the end-of-course assessment. This process uses the traditional assessment method of examination paper, which consists of objective questions. When designing the examination paper, each question is composed of text and identification, and students choose the corresponding identification according to the requirements of the question. Teachers set up questions based on the specific conditions of the laboratory, and students learn about the laboratory's specifications through experiment courses.

\section{CONCLUSION}

In the face of the current situation of diversified access to information by contemporary college students, in view of the problems in the laboratory safety education curriculum of art colleges, the concept of "identification" and the laboratory safety course are combined to mobilize the involvement of students and improve the teaching effect. Compared with traditional course teaching, such a move has the advantages of visualizing the text system, virtualizing the teaching environment, and decomposing the experiment steps, which is both intuitive and visible, well combining the disciplinary advantages of art colleges, providing a reference for the development of laboratory courses, and laying a good foundation for cultivating all-round and comprehensive artistic talents.

\section{AUTHORS' CONTRIBUTIONS}

This paper is independently completed by Lingtao Liu. 


\section{REFERENCES}

[1] Qifeng Zhang, Songyang Wang. Research Summary and Promotion Path of Scientific Research of University Laboratory Safety Education [J]. Journal of Research in Vocational Education, 2020,2(11):

[2] Mowafaq Alsaggar, Monther Al Atoum. The Image as a method of teaching in Art education [J]. Gazi University Journal of Science Part B: Art Humanities Design and Planning, 2019(2):

[3] Yang Rui. Difficulties and Solutions of Laboratory Safety Education in Colleges and Universities [J]. Canadian Social Science, 2021,17(3):

[4] Fred Leland. Rush. Appreciating Susan Sontag [J]. Philosophy and Literature. 2009 (1)

[5] Jezia Zakraoui, Moutaz Saleh, Jihad Ja'am. Text-to-picture tools, systems, and approaches: a survey [J]. Multimedia Tools and Applications, 2019,78(16):Jezia Zakraoui, Moutaz Saleh, Jihad Ja'am. Text-to-picture tools, systems, and approaches: a survey [J]. Multimedia Tools and Applications, 2019,78(16):

[6] Hongyan Quan, Chenhui Li. An Effective Practice Method of Visual Case Teaching [J]. Science Innovation, 2021,9(2):

[7] Neil C. M. Brown. Studies in Philosophical Realism in Art, Design and Education [M]. Springer, Cham: 